\title{
Decay of Correlations in Ferromagnets
}

\author{
Barry Simon \\ Departments of Mathematics and Physics, Princeton University, Princeton, New Jersey 08544
}

(Received 7 December 1979)

\begin{abstract}
Some new correlation inequalities are described which bound large-distance behavior of correlations in ferromagnets from above by correlations at intermediate distances. Among applications are (1) an inequality, $\eta<1$, on the decay of correlations at the critical point; (2) an inequality $\chi \geqq \operatorname{coth}\left(\frac{1}{2} m\right)$ relating the zero-field susceptibility and the mass gap in a nearest-neighbor ferromagnet; (3) a finite algorithm for rigorously computing a sequence of upper bounds guaranteed to converge to the true transition temperature in Ising ferromagnets.
\end{abstract}

In this note, I wish to describe some new ${ }^{1}$ correlation inequalities in ferromagnetic models which have the effect of bounding very-large-distance correlations from above by correlations at intermediate distances. Many of the more interesting applications involve the use of these inequalities to get lower bounds on intermediatedistance behavior which have the effect of showing that the threshold singularities in various spectral weights cannot be too soft. In models with reflection positivity, ${ }^{2}$ the new inequalities are complementary to consequences of reflection positivity which typically bound long-distance behavior from below. ${ }^{3}$ Various technical details and further consequences of these ideas will appear elsewher $\mathrm{e}^{4}$ and additional developments motivated by this in forthcoming papers by $\mathrm{Lieb}^{5}$ and by Aizenman and Simon. ${ }^{6}$

To explain the basic idea, suppose that $\left\langle s_{\alpha} s_{\beta}\right\rangle$ is the two-point function in a one-component ferromagnet and suppose we have an inequality of the form

$$
\left\langle s_{\alpha} s_{\gamma}\right\rangle \leqslant \sum_{\alpha=\delta \in B} a(\alpha-\delta)\left\langle s_{\delta} s_{\gamma}\right\rangle
$$

In (1), $B$ is some set of sites, $\{\lambda\}$ with $|\lambda|<R$ and (1) is supposed to hold whenever $|\gamma-\alpha|>R$. We clain that $\left\langle s_{\alpha} s_{\beta}\right\rangle$ has exponential falloff so long as $a \geqslant 0$ so that

$$
\sum_{\alpha \in B} a(\alpha) \equiv \Gamma<1
$$

For, if $|\alpha-\gamma|>n R$, iterate (1) $n$ times and find that

$$
\left\langle s_{\alpha} s_{\gamma}\right\rangle \leqslant \Gamma^{n} \max _{\alpha, \beta}\left|\left\langle s_{\alpha} s_{\beta}\right\rangle\right|
$$

so that

$$
\left\langle s_{\alpha} s_{\gamma}\right\rangle \leqslant \max _{\alpha}\left|\left\langle s_{\alpha}{ }^{2}\right\rangle\right| \exp \left[R^{-1} \ln (\Gamma)|\alpha-\gamma|\right] .
$$

One can improve ${ }^{4}$ the value of the mass gap in (3) but the main point ${ }^{7}$ is that (1) and (2) yield a mass gap.

Equations of the form (1) hold in the following cases:

(A) For spin- $\frac{1}{2}$ Ising model with $B$ the set of spins coupled to $\alpha=0$ and $a(\alpha-\delta)=\tanh \left(\beta J_{\alpha \delta}\right)$, (1) is exactly Griffiths's third inequality. ${ }^{8}$

(B) By a graphical analysis, ${ }^{4}$ we have succeeded in proving

$$
\left\langle s_{\alpha} s_{\gamma}\right\rangle \leqslant \sum_{\delta \in B}\left\langle s_{\alpha} s_{\delta}\right\rangle\left\langle s_{\delta} s_{\gamma}\right\rangle
$$

for any family of pair interacting spin- $\frac{1}{2}$ Ising ferromagnets. Here $B$ is any set with the propperty that for any path from $\alpha$ to $\gamma$ whose links are between spins directly linked in $H$, the path must pass through $B$. For example, in a nearestneighbor model, $B$ can be any set whose removal disconnects $Z^{d}$ in such a way that $\alpha$ and $\gamma$ are in distinct pieces.

(C) $\mathrm{Lieb}^{5}$ has extended our proof of (4) to prove the analog of (4) with $\left\langle s_{\alpha} s_{\delta}\right\rangle$ replaced by $\left\langle s_{\alpha} s_{\delta}\right\rangle^{\prime}$ where $\langle\cdots\rangle\rangle^{\prime}$ is the expectation value in the system where we keep only interactions between spins "inside" $B$ and between spins inside $B$ and in $B .{ }^{9}$ When $B$ is the set of "neighbors" of $\alpha$, Lieb's improvement is precisely Griffiths's third inequality.

(D) By Griffiths's trick, ${ }^{10}$ Eq. (4) and Lieb's improvement hold for (suitably normalized ${ }^{11}$ ) spin$S$ Ising spins.

(E) Let $A, B$ be a breakup of all spins into two disjoint pieces with $\alpha \in A, \gamma \in B$. Then using Griffiths's second inequality ${ }^{12}$ and the Lebowitz inequality, ${ }^{13}$ one can prove that ${ }^{4}$

$$
\begin{aligned}
\left\langle s_{\alpha} s_{\gamma}\right\rangle \leqslant \sum_{\delta \in A, \delta^{\prime} \in B} \beta J_{\delta \delta^{\prime}} & \left(\left\langle s_{\alpha} s_{\delta}\right\rangle\left\langle s_{\delta^{\prime}} s_{\gamma}\right\rangle\right. \\
& \left.+\left\langle s_{\alpha} s_{\delta^{\prime}}\right\rangle\left\langle s_{\delta} s_{\gamma}\right\rangle\right) .
\end{aligned}
$$

This inequality and its proof holds for many single-spin distributions including lattice $\varphi^{4}$ field theories and also for plane rotors. 
(F) Using the local Ward identities of Dreissler et al. ${ }^{14}$ Aizenman and Simon ${ }^{6}$ have proven, for $D$-component models of isotropically coupling unit spins,

$$
\left\langle\overrightarrow{\mathbf{s}}_{\alpha} \cdot \overrightarrow{\mathbf{s}}_{\gamma}\right\rangle \leqslant \beta \sum_{\delta} D^{-1} J_{\alpha \delta}\left\langle\overrightarrow{\mathbf{s}}_{\delta} \cdot \overrightarrow{\mathbf{s}}_{\gamma}\right\rangle,
$$

and, for $D=2,3$, and 4 and $A, B$ as in case (E),

$$
\left\langle\overrightarrow{\mathrm{s}}_{\alpha} \cdot \overrightarrow{\mathrm{s}}_{\gamma}\right\rangle \leqslant \beta \sum_{\delta, A_{,} \delta^{\prime} \in B} D^{-1} J_{\delta \delta^{\prime}}\left\langle\overrightarrow{\mathrm{s}}_{\alpha} \cdot \overrightarrow{\mathrm{s}}_{\delta}\right\rangle\left\langle\overrightarrow{\mathrm{s}}_{\delta^{\prime}} \cdot \overrightarrow{\mathrm{s}}_{\gamma}\right\rangle .
$$

Here are some of the applications of these inequalities:

(1) Mass gap for "large" temperatures.-Generalizing the idea of Krinsky and Emery, ${ }^{1}$ I get a mass gap at sufficiently large temperatures. For example, by Eq. (6), I get a mass gap ${ }^{15}$ in the $D$-component model if $\beta \sum J_{0 \delta}<D$. In particular, specializing to the nearest-neighbor twodimensional rotor, I get an upper bound on the Kosterlitz-Thouless transition temperature $T_{0},{ }^{16}$

$$
\left(T_{0}\right)^{-1} \geqslant 0.5 \text {. }
$$

(2) $\eta<1$.-By definition, at the critical point, there is no mass gap. Thus, by Eqs. (2)-(4), we must have that $\sum_{\alpha}\left\langle s_{0} s_{\alpha}\right\rangle \geqslant 1$ for any set of $\alpha$ 's surrounding 0 in the nearest-neighbor case. If ${ }^{17}$ $\left\langle s_{0} s_{\alpha}\right\rangle \sim C|x|^{-(d-2+\eta)}$, this implies that $\eta<1$. This improves the bound $\eta<2$ of Glimm and Jaffe. ${ }^{18}$ With use of $(E)$ this extends to large families of models and it also extends to any finite-range pair ferromagnetic interaction.

(3) Lower bound on $f$. - Let $f(i)=\sum_{\alpha_{1}=i}\left\langle s_{0} s_{\alpha}\right\rangle$. Then by (4),

$$
f(i+j) \leqslant f(i) f(j),
$$

from which one easily concludes that ${ }^{4}$

$$
f(i) \geqslant e^{-m|i|} \text {. }
$$

In the nearest-neighbor case, using reflection positivity, one finds that ${ }^{4}$

$$
\left\langle\sigma_{0} \sigma_{(x, 0, \ldots, 0)}\right\rangle \geqslant 2^{-d}(2|x|+1)^{-d} e^{-m|x|} .
$$

(4) Lower bound on $\chi$.-Summing (8) on $i$ yields

$$
\chi \geqslant \operatorname{coth}\left(\frac{1}{2} m\right) \text {, }
$$

where $\chi$ is the zero-field susceptibility. This yields a new proof of the known result ${ }^{18}$ that $\chi$ $\rightarrow \infty$ at the critical point and a new inequality on critical exponents $\gamma \geqslant \nu$.

(5) Bound on $Z$.-In the nearest-neighbor case, (8) implies that there is a nonzero residue in the single-particle pole, i.e., the quantity $Z$ of Glimm and Jaffe ${ }^{18}$ is bounded from below by $\sinh m$.
The last two applications are consequences of Lieb's improved version (C) above.

(6) Finite algorithm for $\beta_{c} \cdot{ }^{19}$-Consider the nearest-neighbor $d$-dimensional cubic Ising model. Let $\Lambda_{n}$ be set consisting of the cube of side $2 n+1$ together with its nearest neighbors. Let $\partial \Lambda_{n}$ be the set of these outer neighbors and let $f_{n}(\beta)=\sum_{\alpha \in \partial \Lambda_{n}}\left\langle s_{0} s_{\alpha}\right\rangle_{n}$ with $\langle\cdots\rangle_{n}$ the finite-volume states in $\Lambda_{n}$ with free boundary conditions. Determine $\beta_{n}$ by $f_{n}\left(\beta_{n}\right)=1$. Then ${ }^{4}$ it is not hard to see that $\beta_{n} \rightarrow \beta_{c}$, the inverse critical temperature, ${ }^{14}$ as $n \rightarrow \infty$. Each $\beta_{n}$ is, in principle, finitely computable. Unfortunately, the convergence seems to be very slow. ${ }^{4}$ For example in $d=2$, where the exact answer is $\tanh \beta_{c}=0.414 \ldots, \mathrm{I}$ have found that $\tanh \beta_{0}=0.25$, the mean-field value and $\tanh \beta_{1}=0.31328 \ldots$ by exact calculation. ${ }^{20}$ Exact calculations of $\beta_{n}$ for $n \geqslant 2$ seem impractical, but rigorous bounds may be computable so that the method may be practical, but in any event, I emphasize that one can, in principle, compute sequences of upper bounds that converge to the right answer.

(7) Vanishing of the critical point.-U Using Lieb's improved version, I have recovered the McBryan-Rosen result ${ }^{21}$ that as $\beta \rightarrow \beta_{0}{ }^{-}, m \rightarrow 0^{+}$in the nearest-neighbor Ising model.

This work originated in an attempt to understand some new results of Dobrushin ${ }^{22}$ which among other things deduce that sufficiently rapid power falloff of dependence on boundary conditions implies exponential falloff. Some observations of R. Israel were invaluable in putting me on the right track towards Eq. (4). Since then, I have benefitted from discussions with M. Aizenman, J. Bricmont, J. Lebowitz, E. Lieb, A. So$\mathrm{kal}$, and T. Spencer. The author acknowledges partial support of the National Science Foundation under Grant No. MCS-78-01885.

\footnotetext{
${ }^{1}$ These inequalities are extensions of Griffith's third inequality [R. Griffiths, Commun. Math. Phys. $\underline{6}, 121$ (1967)] which is known to yield mass gaps at large temperatures [see S. Krinsky and V. Emery, Phys. Lett. 50A, 235 (1974)]. What is really new here is the possibility of obtaining information at and near the critical point.

${ }^{2}$ For extensive discussion of reflection positivity including earlier references, see J. Fröhlich et al ., Commun. Math. Phys. 62, 1 (1978).

${ }^{3}$ For example, if $g(j)$ is the two-point function $\left\langle\sigma_{\alpha} \sigma_{\beta}\right\rangle$ with $\alpha-\beta=(j, 0, \ldots, 0)$ in a nearest-neighbor Ising model, reflection positivity implies that $g(j)$
} 
$\leqslant e^{-m|j|}$ with $m=\lim _{j \rightarrow \infty}(-j)^{-1} \ln [g(j)]$.

${ }^{4} \mathrm{~B}$. Simon, to be published.

${ }^{5} \mathrm{E}$. Lieb, to be published.

${ }^{6} \mathrm{M}$. Aizenman and B. Simon, to be published.

${ }^{7}$ In a concrete situation, this was already realized

by Krinsky and Emery, Ref. 1.

${ }^{8}$ See Ref. 1.

${ }^{9}$ More precisely, consider all paths from $\alpha$ to $\gamma$ with links of the allowed type. Consider the part of the path from $\alpha$ until the first meeting with the set $B .\langle\cdots\rangle^{\prime}$ has all interactions occurring in these parts of paths.

${ }^{10}$ R. Griffiths, J. Math. Phys. 10, 1559 (1969).

${ }^{11}$ The normalization is that the difference of successive values be 2 . Thus, in (4) we are not able to take the $S \rightarrow \infty$ limit and attain spins uniformly distributed in $[-1,1]$. But method (E) does work for such spins.

${ }^{12}$ R. Griffiths, J. Math. Phys. $\underline{8}, 478,484$ (1967).

${ }^{13}$ J. Lebowitz, Commun. Math. Phys. 35, 87 (1974).

${ }^{14}$ W. Dreisler, L. Landau, and J. Fernando-Perez, J. Stat. Phys. 20, 123-162 (1979).

${ }^{15}$ It is already known Isee B. Simon (J. Stat. Phys., to be published)] that there is no spontaneous magnetization in this case.

${ }^{16}$ Normalized so that at $T=1$ each pair of nearest neighbors has weight 1 in the Hamiltonian. Using different methods, J. Fröhlich and T. Spencer (private communication) have obtained the same bound.

${ }^{17}$ We emphasize that bounds like (8) and (9) hold independent of any hypothesis on the form of the falloff.

${ }^{18} \mathrm{~J}$. Glimm and A. Jaffe, Commun. Math. Phys. 51, 1 (1976), and 52, 263 (1977).

${ }^{19}$ Defined so that $\beta_{c}=\sup \{\beta \mid$ there is a mass gap at $\beta\}$.

${ }^{20}$ This compares unfavorably with Fisher's bound [M. Fisher, Phys. Rev. 162, 480 (1967)] of $\tanh \beta_{c}$ $\geqslant 0.37$ and even with the bound $\tanh \beta_{c} \geqslant 0.33$ which is obtained trivially with Fisher's method.

${ }^{21} \mathrm{O}$. McBryan and J. Rosen, Commun. Math. Phys. 51, 97 (1967).

${ }^{22}$ R. L. Dobrushin, unpublished. I am grateful to R. Israel, M. Ainzenman, and J. Bricmont for communicating Dobrushin's results to me.

\title{
Periodic Oscillations of the Frequency-Dependent Photoelectric Cross Sections of Surface States: Theory and Experiment
}

\author{
Steven G. Louie ${ }^{(a)}$ \\ Bell Laboratories, Murray Hill, New Jersey 07974 \\ and \\ P. Thiry, R. Pinchaux, and Y. Petroff \\ Laboratoire pour l' Utilisation du Rayonnement Electromagnétique, Orsay, France, and Laboratoire de Physique \\ des Solides, Université Pierre et Marie Curie, 75230 Paris Cédex 05, France \\ and \\ D. Chandesris and J. Lecante \\ Laboratoire pour l' Utilisation du Rayonnement Electromagnétique, Orsay, France, and Service de Physique \\ Atomique, Commisariat à L'Energie Atomique, 91190 Gif-sur-Yvette, France \\ (Received 27 September 1979)

\begin{abstract}
High-resolution angle-resolved photoemission spectroscopy is employed to study surface states on the $\mathrm{Cu}(111)$ surface, with use of synchrotron radiation in the energy range $18 \leqslant \hbar \omega \leqslant 120 \mathrm{eV}$. The results reveal a novel periodic oscillatory behavior in surface-state emission intensity which leads to identification of additional new surface states. A spectral decomposition theory is proposed to explain the physical origin of the oscillations. It describes the measured intensity profiles and predicts that the oscillations are universal for all surface states.
\end{abstract}

The purpose of this paper is to report the experimental observation and a theoretical explanation of periodic oscillations as a function of photon energy in the photoelectric cross sections of surface states.
Recently angle-resolved photoemission spectroscopy has been actively employed for direct determination of the energy-band dispersion ( $E$ vs $\vec{k}$ ) of many crystals. These measurements have been done mainly at low photon energies ${ }^{1}$ 